\title{
A Prospective Study of Quantitative SPECT/CT for Evaluation of Lung Shunt Fraction Before SIRT of Liver Tumors
}

\author{
Helmut Dittmann ${ }^{1}$, Daniel Kopp ${ }^{1}$, Juergen Kupferschlaeger ${ }^{1}$, Diana Feil ${ }^{1}$, Gerd Groezinger ${ }^{2}$, Roland Syha ${ }^{2}$, \\ Matthias Weissinger ${ }^{1}$, and Christian la Fougère ${ }^{1}$ \\ ${ }^{1}$ Department of Nuclear Medicine and Clinical Molecular Imaging, University of Tuebingen, Tuebingen, Germany; and ${ }^{2}$ Department \\ of Diagnostic and Interventional Radiology, University of Tuebingen, Tuebingen, Germany
}

The lung shunt fraction (LSF) is estimated using ${ }^{99 m}$ Tc-macroaggregated albumin ( $\left.{ }^{99 m} \mathrm{Tc}-\mathrm{MAA}\right)$ imaging before selective internal radiotherapy (SIRT) of the liver to reduce the risk of pulmonary irradiation. Generally, planar scans are acquired after injection of ${ }^{99 m T c-M A A}$ into the hepatic artery. However, the validity of this approach is limited by differences in attenuation between liver and lung tissue as well as inaccurate segmentation of the organs. The aim of this study was to evaluate quantitative SPECT/CT for LSF assessment in a prospective clinical cohort. Methods: Fifty consecutive patients intended to undergo SIRT were imaged within $1 \mathrm{~h}$ after injection of 99mTc-MAA using a SPECT/CT y-camera. Planar scans of the lung and liver region were acquired in anterior and posterior views, followed by SPECT/CT scans of the thorax and abdomen. Emission data were corrected for scatter, attenuation, and resolution recovery using dedicated software. To quantify the radioactivity concentration in the lung, liver, urinary bladder and remainder of the thoracoabdominal body, volumes of interest were defined on the SPECT/CT images. ${ }^{99 m T c-M A A ~ c o n c e n t r a t i o n s ~ w e r e ~ c a l c u l a t e d ~ a s ~}$ percentage injected dose (\%ID). Results: Mean 99mTc-MAA uptake in liver and lung accounted for only $79 \%$ ID, whereas $13.1 \%$ ID was present in the remainder of the body. In all patients, LSF as calculated from planar scans accounted for a median of $6.8 \%$ (range, $3.4 \%-32.3 \%$ ), whereas the SPECT/CT quantitation revealed significantly lower LSF estimates, at a median of $1.9 \%$ (range, $0.8 \%$ $15.7 \%)(P<0.0001$, Wilcoxon test). On the basis of planar imaging, dose reduction or even contraindications to SIRT had to be considered in 10 of 50 patients, as their LSF was calculated at $10 \%$ or more. In contrast, SPECT/CT quantitation showed substantial shunting in only 2 of the 50 patients. Conclusion: Quantitative SPECT/CT reveals that the LSF is considerably lower than shown on planar imaging. Thus, the resulting dose to the lung parenchyma may be less than conventionally assumed. However, the safety of the SPECT/CT-derived dose range will have to be evaluated.

Key Words: radioembolization; SPECT/CT; lung shunt; SIRT; TcMAA

J Nucl Med 2018; 59:1366-1372

DOI: 10.2967/jnumed.117.205203

Received Nov. 14, 2017; revision accepted Jan. 5, 2018.

For correspondence or reprints contact: Helmut Dittmann, University of Tuebingen, Otfried-Mueller-Strasse 14, 72076 Tuebingen, Germany.

E-mail: helmut.dittmann@med.uni-tuebingen.de

Published online Jan. 25, 2018.

COPYRIGHT (C 2018 by the Society of Nuclear Medicine and Molecular Imaging.
O (SIRT), also termed radioembolization using ${ }^{90}$ Y-loaded microspheres, is an increasingly performed treatment for patients with unresectable primary tumors and metastases of the liver $(1,2)$. SIRT has been shown to be safe and well tolerated in most patients (3), but complications may arise when ${ }^{90}$ Y-microspheres bypass the liver vascular system because of arteriovenous shunts. Although any gastrointestinal shunt is regarded as a contraindication to SIRT, hepatopulmonary shunting is accepted if the resulting irradiation dose to the lung is considered tolerable $(4,5)$. A lung dose limit of $30 \mathrm{~Gy}$, as established for external-beam irradiation, is regarded as acceptable, and up to $50 \mathrm{~Gy}$ in total have been judged tolerable for multiple SIRT cycles (6). On the other hand, pulmonary toxicity after SIRT has been described in distinct clinical cases with excessive lung shunting $(7,8)$.

Before SIRT is performed, gastrointestinal shunt vessels may be identified on diagnostic angiography and occluded or avoided by altering the catheter position (9). However, angiography is not suitable for evaluating lung shunting or for safely excluding gastrointestinal shunts. Thus, therapeutic microsphere distribution is simulated by ${ }^{99 \mathrm{~m}} \mathrm{Tc}-\mathrm{macroaggregated}$ albumin ( ${ }^{99 \mathrm{~m} T c-M A A)}$ scintigraphy $(7,10)$. Previous studies $(11,12)$ have shown that SPECT/ CT improves the detection of gastrointestinal shunts that might be missed on planar ${ }^{99 \mathrm{~m}} \mathrm{Tc}-\mathrm{MAA}$ imaging. In contrast to gastrointestinal shunts, interpretation of radioactivity accumulation in the lungs is not a yes-or-no question. Thus, additional quantitation of the lung shunt fraction (LSF) through image analysis is needed to decide whether irradiation of the lung might be tolerable $(6,13,14)$.

So far, LSF has been analyzed in a semiquantitative manner by defining regions of interest (ROIs) on planar scans (7). Nonetheless, quantification based on planar scans is limited by the lower attenuation of lung tissue than of liver tissue. Even more limiting is that ${ }^{99 m}$ Tc-MAA uptake in the liver dome might add to the radioactivity attributed to the basal lung area. SPECT/CT may improve accuracy because the acquired CT scan enables correction for attenuation of $\gamma$-emission. Also, additional corrections have been shown to allow for absolute quantification similar to that used in PET imaging (15-17). Finally, the use of 3-dimensional images enables more accurate segmentation of liver and lung areas.

The first studies with quantitative SPECT/CT confirmed an overestimation of LSF on planar imaging (18-20). To compensate 
for potential liver activity misplacement, investigators excluded the basal lung areas from LSF estimates $(18,19)$. The drawback of this approach is that the actual LSF might be somewhat underrated.

Therefore, the aim of our study was to prospectively evaluate quantitative ${ }^{99 \mathrm{~m}}$ Tc-MAA SPECT/CT for LSF estimation in patients intended to undergo SIRT. In view of the possible shortcomings of quantitative measurements, we addressed artifacts caused by liver overspill and mismatch between CT and SPECT scans.

\section{MATERIALS AND METHODS}

\section{Phantom Study}

To validate SPECT/CT quantification, an anthropomorphic torso phantom (Data Spectrum Corp.) was used. The phantom consisted of liver and lung inserts against a cold background. The ${ }^{99 \mathrm{~m} T c-p e r t e c h n e-}$ tate activity concentration in the lung insert was adjusted to simulate a LSF of approximately $10 \%$. Images were acquired with a dual-detector SPECT/CT camera (Discovery 670 Pro; GE Healthcare). A SPECT scan was acquired using a $128 \times 128$ matrix, 30 steps, and a 120 -s acquisition time per step, followed by a CT scan $(60 \mathrm{~mA}, 120 \mathrm{kV}, 2.5-\mathrm{mm}$ slice thickness) for attenuation correction and anatomic mapping. Images were reconstructed with an ordered-subset expectation maximization iterative protocol (4 iterations, 10 subsets) without pre- or postfiltering. The reconstructed data were then coregistered with the CT images on a dedicated workstation (Xeleris 3; GE Healthcare).

\section{Patient Population}

Fifty consecutive patients intended to receive SIRT were included. All patients had either histologically proven primary liver tumors or metastases from extrahepatic tumors and were not eligible for surgery. The clinical characteristics of the patients are in Table 1. The institutional review board approved this study (decision 747/2014BO1), and all subjects gave written informed consent. The decision on whether to

TABLE 1

Patient Characteristics

\begin{tabular}{|lccc}
\hline & All & Male & Female \\
\hline Patients $(n)$ & 50 & 31 & 19 \\
\hline BMI & & & \\
\hline Mean & 26 & 27 & 24 \\
\hline Range & & $20-64$ & $19-33$ \\
\hline Age & & & \\
\hline Mean & 66 & 65 & 67 \\
\hline Range & & $44-80$ & $39-81$ \\
\hline Histology ( $n$ ) & & & \\
\hline Hepatocellular carcinoma & 15 & 10 & 5 \\
\hline Colorectal carcinoma & 13 & 8 & 5 \\
\hline Cholangiocellular carcinoma & 11 & 6 & 5 \\
\hline Ocular melanoma & 4 & 3 & 1 \\
\hline Pancreatic cancer & 3 & 2 & 1 \\
\hline Cervical cancer & 1 & & 1 \\
\hline Squamous cell carcinoma & 1 & 1 & \\
\hline Midgut neuroendocrine tumor & 1 & 1 & \\
\hline Breast cancer & 1 & & 1 \\
\hline & & & \\
\hline
\end{tabular}

use SIRT treatment was made by an interdisciplinary tumor board at the local comprehensive cancer center.

\section{Angiography}

A robotic angiographic suite (Artis Zeego Q, VE 40 A; Siemens) was used throughout. At first, a superior mesenteric arteriogram was performed to detect accessory or replaced hepatic arteries arising from the superior mesenteric artery. Then, selective right and left hepatic angiography was performed using a 2.7-French microcatheter system (Progreat; Terumo) to evaluate variants of hepatic anatomy and subsequent prophylactic embolization of extrahepatic vessels such as the right gastric, gastroduodenal, or falciform artery.

\section{9mTc-MAA Imaging}

Perchlorate was administered before ${ }^{99 \mathrm{~m}} \mathrm{Tc}-\mathrm{MAA}$ injection to exclude gastric uptake of free ${ }^{99 \mathrm{~m}} \mathrm{Tc}$. Patients received $128 \pm 38 \mathrm{MBq}$ of ${ }^{99 \mathrm{~m}} \mathrm{Tc}-\mathrm{MAA}$ into the arterial branches. Within $1 \mathrm{~h}$ from ${ }^{99 \mathrm{~m}} \mathrm{Tc}-\mathrm{MAA}$ injection, planar images of the lung and liver area were acquired in anterior and posterior views (5-min acquisition, $128 \times 128$ matrix) followed by SPECT/CT. During SPECT, patients were asked to use shallow breathing to minimize respiratory motion.

The SPECT acquisition parameters were 2 fields of view covering thorax and abdomen, a $128 \times 128$ matrix, 30 steps, and a 15 -s acquisition per step. Finally, a diagnostic CT scan (dose-length product, 222-498 mGy-cm, $120 \mathrm{kV}, 2.5-\mathrm{mm}$ slice thickness) was acquired. SPECT images were reconstructed and coregistered with $\mathrm{CT}$ as described above.

To optimize the match between the CT and SPECT acquisitions, patients were asked to use shallow breathing for about $1 \mathrm{~min}$ before the CT acquisition began and then to stop breathing at the baseline level during the acquisition. This procedure was performed because deep inspiration during CT data acquisition comes with an underestimation of attenuation in the cranial part of the liver. Thus, liver activity may be underrated. On the other hand, deep expiration would lead to misplacement of the liver dome into the lung area, leading to possible overcorrection of basal lung counts. Both artifacts may lead to an exaggerated LSF.

\section{Quantification}

To quantify LSF on planar scans, ROIs for the lungs and the liver were defined in anterior and posterior views. Separate ROIs were generated for the left and right lung lobes, omitting the mediastinum and heart. A gap of approximately $1 \mathrm{~cm}$ was left between the basal lung and the liver to limit overspill of liver activity into the lung ROIs. The planar LSF was then computed using the following equations:

$$
\begin{aligned}
\text { Geometric mean (lung or liver }) & \\
= & \sqrt{ }(\text { counts anterior view } \times \text { counts posterior view }) \\
\operatorname{LSF}(\%)= & 100 \times \text { geometric mean lung } /(\text { geometric mean lung } \\
& + \text { geometric mean liver }) .
\end{aligned}
$$

For SPECT/CT quantification, tomographic data were corrected for attenuation, scatter, and resolution recovery by means of a dedicated software algorithm (Evolution; GE Healthcare). Volumes of interest (VOIs) were defined using semiautomatic segmentation (Q.metrics; GE Healthcare). Specifically, a seed point was selected on the transaxial SPECT slices to define radioactivity in the liver, urinary bladder, and remainder of the thoracoabdominal body, whereas CT slices were used to identify the lungs. Initially, the liver volume was rendered by placing a seed point into an area of ${ }^{99 \mathrm{~m} T c-M A A}$ uptake in normal parenchyma to grow a VOI for the liver area (threshold, 10\%-20\% of maximum, adapted to the individual activity distribution). Then, the lung region was identified on the $\mathrm{CT}$ slices (threshold, $-200 \mathrm{HU}$; range, -200 to $-1,000)$. Subsequently, VOIs for the urinary bladder and remainder of 
TABLE 2

SPECT/CT Quantification Using Anthropomorphic Phantom

\begin{tabular}{|c|c|c|c|c|c|c|}
\hline Parameter & Unit & True value & Measured value & Deviation & True volume (mL) & Segmented volume $(\mathrm{mL})$ \\
\hline Left lung & $\mathrm{MBq}$ & 8.7 & $8.7 \pm 0.8^{*}$ & $0 \%$ & 900 & 1,010 \\
\hline Right lung & $\mathrm{MBq}$ & 11.1 & $10.9 \pm 0.6^{\star}$ & $-1.8 \%$ & 1,100 & 1,230 \\
\hline Liver & $\mathrm{kBq} / \mathrm{mL}$ & 116 & $123 \pm 31^{*}$ & $+6 \%$ & 1,160 & 1,120 \\
\hline \multicolumn{7}{|l|}{ LSF } \\
\hline Planar & $\%$ & 11.5 & 15.9 & $+38 \%$ & & \\
\hline SPECT/CT & $\%$ & 11.5 & 12.1 & $+5 \%$ & & \\
\hline *Mean \pm SD. & & & & & & \\
\hline
\end{tabular}

the thoracoabdominal body were defined on the SPECT slices. For comparison, the total lung volume was analyzed from CT without prior liver VOI definition. The radioactivity concentration in the respective regions was calculated as percentage of injected ${ }^{99 \mathrm{~m}} \mathrm{Tc}-\mathrm{MAA}$ dose (\%ID). The SPECT/CT-derived LSF was then calculated using the following equation:

$$
\operatorname{LSF}(\%)=100 \times \% \text { ID lung } /(\% \text { ID lung }+\% \text { ID liver }) .
$$

\section{Dose Calculations}

Estimations of the irradiation dose delivered by ${ }^{90} \mathrm{Y}$-microspheres were based on the simplified MIRD formula (6):

Tissue dose $(\mathrm{Gy})=\operatorname{injected}$ activity $(\mathrm{GBq}) \times 50 /$ tissue mass $(\mathrm{kg})$.

The dose to the lungs could be calculated as described by Ho et al. (10) using the following equation:

Lung dose $(\mathrm{Gy})=$ injected activity $(\mathrm{GBq}) \times \mathrm{LSF} \times 50 /$ lung mass $(\mathrm{kg})$.

To calculate the ${ }^{90}$ Y-activity needed for SIRT, algorithms were used as recommended by the respective microsphere manufacturers $(13,14)$. In our study, resin microspheres (SirSpheres; Sirtex) were used as the standard of care, whereas patients with portal vein thrombosis received less-embolizing glass microspheres.

The activity of the resin microspheres was estimated on the basis of body surface area as a surrogate for the patient's liver volume, using the following formulation:

Injected activity $(\mathrm{GBq})$

$$
=(\text { body surface area }-0.2)+\text { fractional tumor involvement }
$$$$
\times \% \text { treated liver } / 100 \text {. }
$$

According to the recommendations of the manufacturer, Sirtex, the injected activity should be reduced when the LSF is high (13). The

TABLE 3

Radioactivity in Patient Lung, Liver, Urinary Bladder, and Remainder of Thoracoabdominal Body as Determined from 99mTC-MAA SPECT/CT

\begin{tabular}{lccr}
\hline \multicolumn{1}{c}{ Compartment } & Median \%ID & Mean \%ID & SD \\
\hline Lung & 2.5 & 1.4 & 3.0 \\
\hline Liver & 78 & 77.6 & 10.4 \\
Urinary bladder & 1 & 0.9 & 0.9 \\
\hline Remainder of body & 13.4 & 12.2 & 6.7 \\
\hline
\end{tabular}

activity would be decreased by $20 \%$ for a LSF of $10 \%-15 \%$ or by $40 \%$ for a LSF of $15 \%-20 \%$. A LSF above $20 \%$ is regarded as a contraindication to SIRT.

In patients scheduled for SIRT with glass microspheres (TheraSphere glass microspheres; BTG) a liver target dose of 100-120 Gy was chosen (14). To calculate the activity of the glass microspheres, a modified MIRD equation was used:

$$
\begin{aligned}
\operatorname{Liver} \operatorname{dose}(\mathrm{Gy})= & \text { injected activity }(\mathrm{GBq}) \\
& \times(1-(\operatorname{LSF}[\%] / 100)) \times 50 / \text { liver mass }(\mathrm{kg}) .
\end{aligned}
$$

Rather than reducing the dose, as in case of resin microspheres, this formula increases injected activity to compensate for loss due to lung shunting.

The lung irradiation dose resulting from SIRT was then computed considering LSF, liver target area, and, optionally, the individual lung volume. Lung density measurements were not available in this study; thus, the lung density was assumed to be $0.3 \mathrm{~g} / \mathrm{cm}^{3}(21)$.

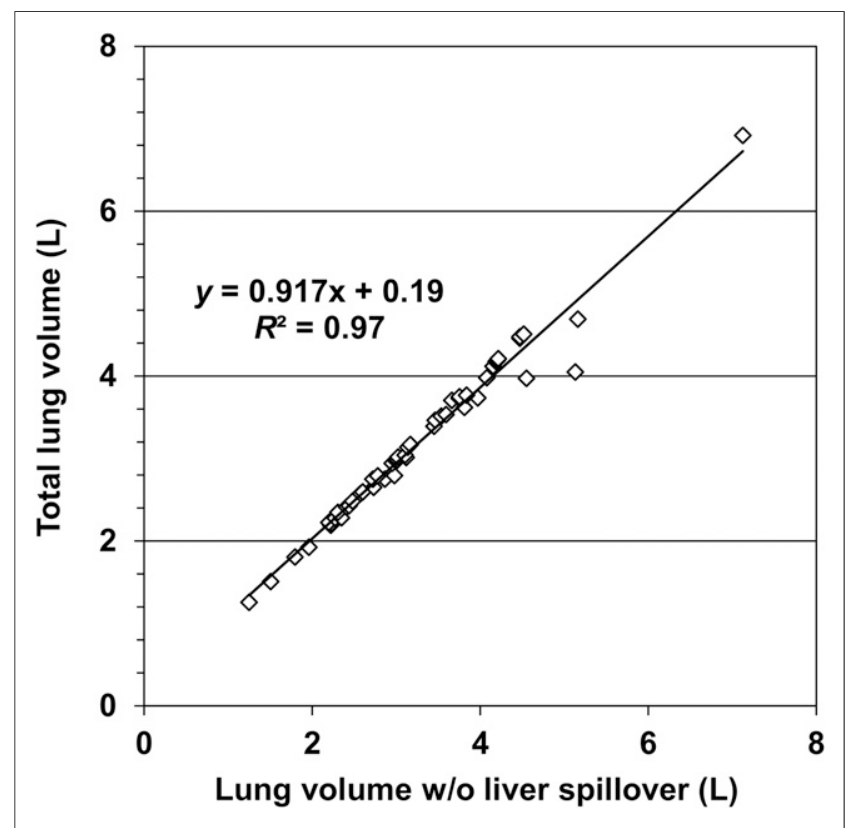

FIGURE 1. Relationship between total lung volume and lung volume after subtraction of liver tissue activity spilling into lung region during SPECT acquisition. 


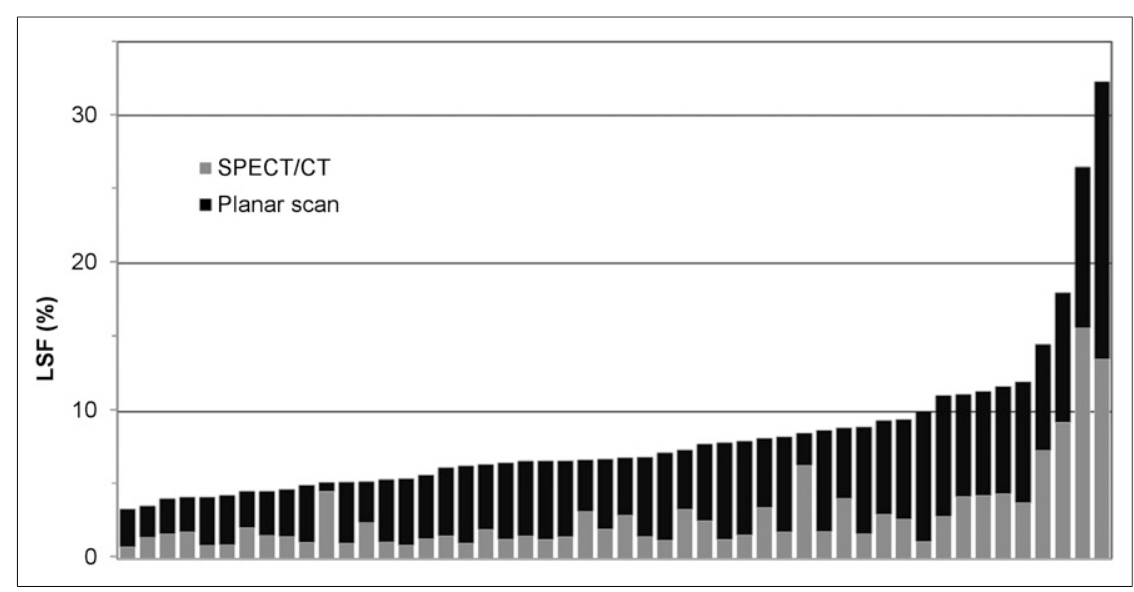

FIGURE 2. Comparison of LSF as calculated from planar imaging and SPECT/CT in 50 patients. Data are presented in incremental order of planar imaging-derived LSF.

\section{Statistics}

The Wilcoxon signed-rank test (2-tailed) was used to compare planar imaging-based and SPECT/CT-based LSF using Excel software (Microsoft Corp.). An $\alpha$-level of 0.05 was considered significant.

\section{RESULTS}

\section{Phantom Study}

Using SPECT/CT, the calculated ${ }^{99 m}$ Tc-MAA deposition in the lung inserts exactly matched the calibrated activity (Table 2). The total lung volume was overestimated by approximately $10 \%$ by the segmentation algorithm. Liver activity was slightly overestimated, whereas the organ volume was measured precisely. As a result, LSF could be quantified with only marginal deviation from the true lung radioactivity by SPECT/CT. In contrast, ROI analysis of planar scans resulted in a LSF overestimation of approximately $40 \%$.

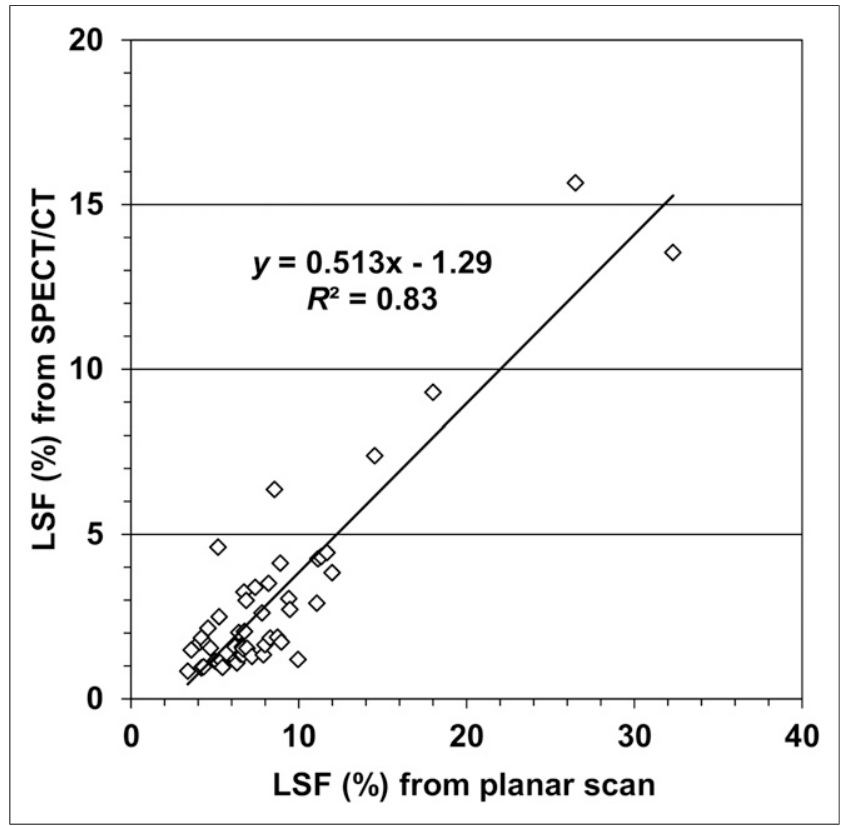

FIGURE 3. Correlation between LSF derived from SPECT/CT and LSF derived from planar imaging.

\section{Patient Study}

On SPECT/CT, mean ${ }^{99 m}$ Tc-MAA uptake was calculated as $1.4 \%$ ID in the lung and as almost $78 \%$ ID in the liver (Table 3 ). Thus, both organs together contained only about $80 \%$ of the decay-corrected radioactivity. The urinary bladder showed prominent activity against the background in 38 of the 50 patients, equivalent to about $1 \%$ ID. The kidney pelvis did not contain noticeable radioactivity in most patients. Focal uptake due to shunts in gastrointestinal regions was observed in 3 patients ( 2 in the small bowel [1.6 and $2.1 \%$ ID] and 1 in the large bowel [9.1\%ID]). Apart from those cases, no pronounced uptake was detected in organs other than liver and lung. Strikingly, $12.2 \%$ ID was present in the remainder of the thoracoabdominal body. Consequently, about $10 \%$ of radioactivity was missing in the SPECT/CT area, most probably because of diffuse distribution in the residual whole body.

To analyze the congruence of CT and SPECT data, total lung volume as measured on $\mathrm{CT}$ alone was compared with residual lung volume after subtraction of liver tissue radioactivity within the lung VOI on the SPECT/CT images. In most patients, there was only a minor difference in volume estimates (mean total lung volume of $3.29 \mathrm{~L}$ vs. liver-subtracted lung volume of $3.21 \mathrm{~L}$ ), indicating a good match between the breath hold during CT and the shallow breathing during SPECT $\left(R^{2}=0.97\right)$ (Fig. 1). In only 6 of the 50 patients did total lung volume exceed the liver activitycorrected measure by more than 5\% (range, 5.1\%-21.1\%).

Median LSF was determined to be $6.8 \%$ based on the planar ${ }^{99 m}$ Tc-MAA scans (mean, 8.3 ; range, $3.4 \%-32.3 \%$ ) but only $1.9 \%$ based on the SPECT/CT scans (mean, 2.9; range, 0.8\%-15.7\%). The planar imaging-derived estimation of LSF was significantly higher in all 50 patients $(P<0.0001)$, resulting in an average LSF that was 3.6 times higher than for the SPECT/CT-based estimation (Fig. 2). Overall, there was a strong correlation $\left(R^{2}=0.83\right)$ in the LSFs calculated by the two approaches (Fig. 3). However, the individual difference between the two estimates was variable, especially in patients exhibiting low LSFs. Using planar imaging data, the LSF was calculated to be $10 \%$ or more in 10 of the 50 patients (Table 4; Fig. 2). In particular, 2 individuals showed an LSF in excess of $20 \%$, suggesting a contraindication to SIRT. In contrast, SPECT/CT quantitation found substantial shunting in only those 2 patients (15.7\% and 13.5\%; Fig. 4) whereas LSF remained below $10 \%$ in all other patients. As a consequence, dose reduction or contraindications to radioembolization had to be considered in $20 \%$ of our patients based on planar imaging-derived LSFs versus less than $5 \%$ based on SPECT/CT-derived LSFs.

\section{Lung Dose Calculation}

Table 4 displays various lung dose estimates for patients with a LSF of $10 \%$ or more on planar imaging. Evidently, the computed lung dose was influenced by the target liver volume, with the doses being lower for patients who were to undergo lobar treatment than for those who were to undergo whole-liver treatment. Relatively high lung doses were calculated for patients scheduled for glass microsphere treatment. In particular, patient 18 (Table 4) would be expected to receive more than $50 \mathrm{~Gy}$ to the lungs or at least $20 \mathrm{~Gy}$ 
TABLE 4

Lung Dose Estimates in the 10 Patients with a LSF of $10 \%$ or More as Determined from Planar $99 \mathrm{mTC}-\mathrm{MAA}$ Imaging

\begin{tabular}{|c|c|c|c|c|c|c|c|c|c|c|}
\hline \multirow[b]{4}{*}{ Patient no. } & \multirow{2}{*}{\multicolumn{2}{|c|}{ LSF (\%) }} & \multirow{4}{*}{$\begin{array}{c}\text { Lung } \\
\text { volume (L) }\end{array}$} & \multirow{4}{*}{$\begin{array}{l}\text { SIRT } \\
\text { area }\end{array}$} & & & \multicolumn{4}{|c|}{ Lung dose ${ }^{\dagger}$ (Gy) } \\
\hline & & & & & \multirow{2}{*}{\multicolumn{2}{|c|}{$\begin{array}{l}{ }^{90} \text { activity } \\
\text { (MBq) }\end{array}$}} & \multicolumn{2}{|r|}{ Planar } & \multicolumn{2}{|c|}{ SPECT/CT } \\
\hline & & SPECT/ & & & & & \multirow[b]{2}{*}{ LSF } & \multirow{2}{*}{$\begin{array}{l}\text { LSF and } \\
\text { lung volume }\end{array}$} & \multirow[b]{2}{*}{ LSF } & \multirow{2}{*}{$\begin{array}{l}\text { LSF and } \\
\text { lung volume }\end{array}$} \\
\hline & Planar & CT & & & Resin & Glass & & & & \\
\hline \multirow[t]{2}{*}{5} & 11.1 & 2.9 & 3.84 & Right lobe & 1.070 & - & 5.9 & 5.3 & 1.6 & 1.4 \\
\hline & & & & & - & 3.600 & 19.9 & 17.7 & 5.2 & 4.6 \\
\hline \multirow[t]{2}{*}{6} & 12.0 & 3.8 & 1.80 & Left lobe & 730 & - & 4.4 & 8.1 & 1.4 & 2.6 \\
\hline & & & & & - & $2.000^{*}$ & 12.0 & 22.3 & 3.8 & 7.1 \\
\hline \multirow[t]{2}{*}{10} & 18.0 & 9.3 & 2.22 & Whole liver & 1.700 & - & 15.3 & 23.4 & 7.9 & 12.1 \\
\hline & & & & & - & 5.000 & 45.0 & 68.8 & 23.2 & 35.5 \\
\hline \multirow[t]{2}{*}{18} & 32.3 & 13.5 & 2.41 & Left lobe & 820 & - & 13.2 & 18.4 & 5.6 & 7.7 \\
\hline & & & & & - & $2.730^{*}$ & 44.1 & 61.2 & 18.5 & 25.7 \\
\hline \multirow[t]{2}{*}{19} & 26.5 & 15.7 & 3.17 & Whole liver & 1.900 & - & 25.2 & 26.3 & 14.9 & 15.6 \\
\hline & & & & & - & 6.920 & 91.7 & 95.9 & 54.2 & 56.6 \\
\hline \multirow[t]{2}{*}{21} & 11.7 & 4.4 & 2.98 & Whole liver & 1.820 & - & 10.6 & 12.7 & 4.0 & 4.8 \\
\hline & & & & & - & 8.490 & 49.6 & 59.5 & 18.9 & 22.6 \\
\hline \multirow[t]{2}{*}{29} & 11.1 & 4.2 & 2.60 & Whole liver & 1.520 & - & 8.5 & 10.9 & 3.2 & 4.2 \\
\hline & & & & & - & 4.480 & 25.0 & 32.2 & 9.5 & 12.3 \\
\hline \multirow[t]{2}{*}{30} & 11.3 & 4.3 & 4.52 & Left lobe & 900 & - & 5.1 & 3.8 & 1.9 & 1.4 \\
\hline & & & & & - & $2.200^{*}$ & 12.5 & 9.3 & 4.7 & 3.5 \\
\hline \multirow[t]{2}{*}{33} & 10.0 & 1.2 & 4.48 & Left lobe & 770 & - & 3.8 & 2.9 & 0.5 & 0.3 \\
\hline & & & & & - & 2.070 & 10.3 & 7.7 & 1.2 & 0.9 \\
\hline \multirow[t]{2}{*}{34} & 14.5 & 7.4 & 3.02 & Whole liver & 1.850 & - & 13.4 & 14.9 & 6.8 & 7.6 \\
\hline & & & & & - & 2.250 & 16.4 & 18.1 & 8.3 & 9.2 \\
\hline
\end{tabular}

as based on SPECT/CT. Notably, consideration of the CT-derived individual lung volume resulted in a $20 \%-45 \%$ increase in lung dose in 4 patients with comparatively low lung volume.

\section{SIRT}

SIRT was performed in 39 of 50 patients within 2 wk from SPECT/CT dosimetry. Individual reasons for not performing SIRT were a noncorrectable gastrointestinal shunt (4 patients: 3 detected on ${ }^{99 m}$ Tc-MAA imaging and 1 newly evolved at repeated angiography), insufficient tumor targeting on ${ }^{99 \mathrm{~m}} \mathrm{Tc}$ MAA SPECT/CT (3 patients), newly evolved extrahepatic disease detected on CT (2 patients), or critically worsened liver function in the interval between simulation and planned SIRT (2 patients). No patient was excluded because of lung shunting. In particular, both individuals with a LSF exceeding $20 \%$ on planar scans (patients 18 and 19; Table 4) received SIRT. Patient 18 had disease only in the left liver and was planned to undergo lobar treatment with glass microspheres. In this patient, we chose to slightly reduce the liver target dose to $100 \mathrm{~Gy}$. Patient 19 received full-dose SIRT of the entire liver using a 2-step approach with a 6-wk interval between treatment of the right and left lobes. The mean length of follow-up after SIRT for all patients was $7.2 \mathrm{mo}$ (range, 3-18 mo), without any signs of pulmonary damage.

\section{DISCUSSION}

The quantification algorithm used in the current study enabled reliable determination of LSF on ${ }^{99} \mathrm{~m}$ Tc-MAA SPECT/CT, whereas planar imaging led to a considerable overestimation. Using an anthropomorphic torso phantom, we demonstrated that liver and lung radioactivity deposition could be accurately quantified by SPECT/CT.

Our clinical evaluation revealed lower LSF estimates based on SPECT/CT in all patients. Remarkably, the inconsistency between planar imaging-derived and SPECT/CT-derived LSF was greatest in patients with a low LSF. This finding might be explained by the higher relative contribution of liver activity near the basal lung area on planar imaging.

Overestimation of LSF was especially relevant in a subgroup of 10 patients for whom planar scan analysis indicated the need for dose reduction or even a contraindication to SIRT, according to current guidelines (4). As based on SPECT/CT, only 2 of these patients had a LSF for which dose modification had to be considered. 


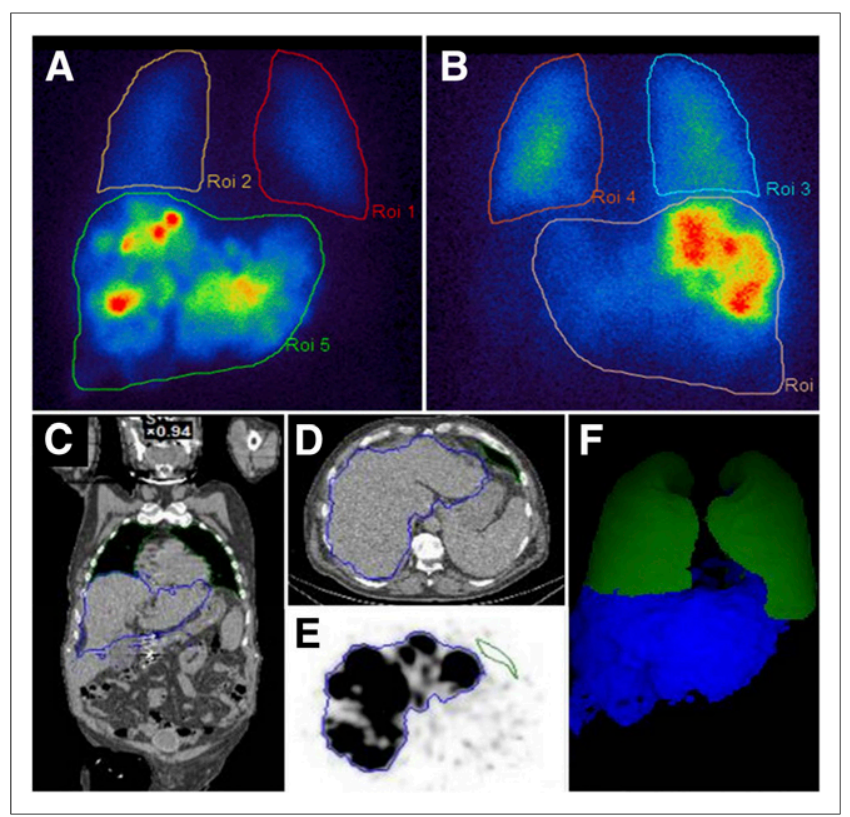

FIGURE 4. High-LSF case (patient 19; Table 4). LSF was estimated at $26.5 \%$ using ${ }^{99 m T C-M A A ~ p l a n a r ~ i m a g i n g ~ a n d ~ a t ~} 15.7 \%$ using ${ }^{99 m T c}-M A A$ SPECT/CT. Shown are anterior (A) and posterior (B) $99 \mathrm{mTC}-\mathrm{MAA}$ planar scans, central coronal CT slice with delineated lung and liver areas (C), transaxial CT scan showing liver and basal lung area (D), corresponding 99mTC-MAA SPECT image (E), and VOls for lung (green) and liver (blue) (F).

These results are in accordance with earlier studies $(17,18,20)$ highlighting overestimation of LSF due to lower attenuation of lung parenchyma on planar imaging. However, another retrospective study (19) reported agreement with the SPECT/CT-derived LSF in some patients.

Aiming to avoid spillover of liver radioactivity into the lung area, previous investigators have either excluded the basal lung (19) or used only the left lung lobe (18) for quantification. The resulting underestimation of lung radioactivity was then compensated for by correcting for the total lung volume as measured by CT. The drawback of these approaches is that they will depend on homogeneous perfusion throughout the lung parenchyma, a requirement that is not necessarily fulfilled (5) since the basal and central lung areas are subject to comparatively higher-level perfusion (22). In our study, we chose to delineate the liver area using its intensive ${ }^{99 m}$ Tc-MAA uptake before definition of all lung parenchyma outside the liver area using CT Hounsfield unit density. In addition, we aimed to acquire the CT images in a way that would allow for attenuation correction of the selective basal lung/liver dome area by asking patients to stop breathing at the baseline level during $\mathrm{CT}$ and to use shallow breathing during SPECT. These measures were communicated meticulously to patients before they underwent SPECT/CT and were usually well tolerated. Because comparison of lung volumes with and without subtraction of liver activity showed no relevant misplacement of the liver into the lung area in most patients, we conclude that this method allows for an acceptable match of SPECT and CT. However, we are aware that an exact definition of the liver area from emission data would depend on a respiration-gated SPECT acquisition.

In addition to LSF, the lung irradiation dose will be influenced by the individual lung mass. Common methodology uses a standard lung mass of $1,000 \mathrm{~g}$, thus neglecting individual variations in lung volume and density $(4,5)$. Conversely, it is well recognized that lung volume varies considerably between individuals whereas density is relatively constant in healthy lungs (23). Similar results have been demonstrated by Kao et al. (19), who used diagnostic CT to estimate lung volume and density in a dedicated lung dosimetry study. Thus, sufficient assessment of the lung irradiation dose should comprise LSF and individual lung volume, ideally complemented by CT densitometry.

In the current study, only about $80 \%$ of the expected radioactivity was in the liver and lungs whereas a considerable amount was present in the remainder of the body. Possible reasons for this finding are gastrointestinal shunts, right-to-left shunting, or disintegration of the radiotracer. Gastrointestinal uptake was, in fact, observed in 3 patients, though accounting for only a minor part of the extrahepatopulmonary radioactivity. In cases of circulation collateral to the lungs, renal parenchyma uptake will be expected (24). Because no kidney uptake was seen in any of our patients, we conclude that activity in the remainder of the body was predominantly due to 99m Tc-MAA disintegration. This finding emphasizes the need for perchlorate blockade to prevent stomach uptake, which might be misinterpreted as gastric shunting (25).

99mTc-MAA has been shown to be unstable in vivo, leading to an increase in LSF and a longer interval between injections and imaging (26). A recent study with planar imaging (27) demonstrated that for SIRT simulation, human serum albumin represents a more stable radiotracer than ${ }^{99 \mathrm{~m}} \mathrm{Tc}-\mathrm{MAA}$. Our study confirmed significant radioactivity that is not retained in the liver or lung compartment as early as $1 \mathrm{~h}$ from ${ }^{99 \mathrm{~m}}$ Tc-MAA injection. Naturally, imaging at an even earlier time might have reduced the disintegrated ${ }^{99 \mathrm{~m}} \mathrm{Tc}-\mathrm{MAA}$ fraction; however, earlier imaging was not practicable because of transfer of patients from the angiography unit. Because planar methodology involves ROI analysis of summed counts originating from the lungs and liver as well as from overlying tissue in the thoracic and abdominal walls, ${ }^{99 \mathrm{~m}} \mathrm{Tc}-$ MAA fragments in the background might have added to the LSF.

Investigators have shown that LSF can be reduced by pretreatment with antiangiogenic agents such as sorafenib (28) and bevacizumab (29) or by interventional techniques (30). Also, there is a growing body of evidence showing that LSF is an independent prognostic factor for patients with liver tumors $(31,32)$. Improved quantification by SPECT/CT will be helpful in analyzing the effects of pretreatment and in exploring the prognostic potential of LSF in patients treated with SIRT.

Our clinical study had some limitations. There was no external gold standard to define LSF independently of ${ }^{99 \mathrm{~m} T c-M A A}$ distribution. A study that compared ${ }^{99 m}$ Tc-MAA with the novel radioembolization agent ${ }^{166} \mathrm{Ho}-$ microspheres showed considerable pulmonary uptake of ${ }^{99 m}$ Tc-MAA but not of ${ }^{166} \mathrm{Ho}$ in some patients (33). The authors concluded that ${ }^{99 \mathrm{~m}} \mathrm{Tc}-\mathrm{MAA}$, because of its considerable content of small-diameter particles $(<20 \mu \mathrm{m})$, might be more prone to arteriovenous shunting than are the therapeutic microspheres, thus exaggerating LSF.

Because of the low incidence of excessive lung shunting, only a few patients with a high LSF could be included in our study. No irradiation damage to the lungs was evident, but the follow-up interval was limited. Thus, late pulmonary toxicities could not be excluded. Hence, the safety information on SIRT in patients with a high LSF on ${ }^{99 m}$ Tc-MAA SPECT/CT has to be considered preliminary. The tolerable lung dose will have to be further evaluated.

\section{CONCLUSION}

SPECT/CT enables quantification of LSF from ${ }^{99 m}$ Tc-MAA scans in patients before SIRT. Because SPECT/CT-based LSF is 
significantly lower than LSF derived from planar imaging, the resulting dose to the lung parenchyma may be less than conventionally assumed. However, the safety of the SPECT/CT-based dose range will have to be evaluated. Our study highlights considerable in vivo instability of ${ }^{99} \mathrm{~m}$ Tc-MAA even if imaging is performed within $1 \mathrm{~h}$ after injection.

\section{DISCLOSURE}

This study was in part funded by a SPECT/CT research grant from GE Healthcare to Christian la Fougère. No other potential conflict of interest relevant to this article was reported.

\section{REFERENCES}

1. Hendlisz A, Van den Eynde M, Peeters M, et al. Phase III trial comparing protracted intravenous fluorouracil infusion alone or with yttrium-90 resin microspheres radioembolization for liver-limited metastatic colorectal cancer refractory to standard chemotherapy. J Clin Oncol. 2010;28:3687-3694.

2. Salem R, Lewandowski RJ, Mulcahy MF, et al. Radioembolization for hepatocellular carcinoma using yttrium-90 microspheres: a comprehensive report of long-term outcomes. Gastroenterology. 2010;138:52-64.

3. Riaz A, Awais R, Salem R. Side effects of yttrium-90 radioembolization. Front Oncol. 2014;4:198.

4. Kennedy A, Nag S, Salem R, et al. Recommendations for radioembolization of hepatic malignancies using yttrium-90 microsphere brachytherapy: a consensus panel report from the Radioembolization Brachytherapy Oncology Consortium. Int J Radiat Oncol Biol Phys. 2007;68:13-23.

5. Salem R, Parikh P, Atassi B, et al. Incidence of radiation pneumonitis after hepatic intra-arterial radiotherapy with yttrium-90 microspheres assuming uniform lung distribution. Am J Clin Oncol. 2008;31:431-438.

6. Salem R, Lewandowski RJ, Gates VL, et al. Research reporting standards for radioembolization of hepatic malignancies. J Vasc Interv Radiol. 2011;22:265-278.

7. Leung WT, Lau WY, Ho SK, et al. Measuring lung shunting in hepatocellular carcinoma with intrahepatic-arterial technetium-99m macroaggregated albumin. J Nucl Med. 1994;35:70-73.

8. Wright CL, Werner JD, Tran JM, et al. Radiation pneumonitis following yttrium90 radioembolization: case report and literature review. J Vasc Interv Radiol. 2012;23:669-674.

9. Theysohn JM, Ruhlmann M, Muller S, et al. Radioembolization with Y-90 glass microspheres: do we really need SPECT-CT to identify extrahepatic shunts? PLoS One. 2015;10:e0137587.

10. Ho S, Lau WY, Leung TW, Chan M, Johnson PJ, Li AK. Clinical evaluation of the partition model for estimating radiation doses from yttrium-90 microspheres in the treatment of hepatic cancer. Eur J Nucl Med. 1997;24:293-298.

11. Hamami ME, Poeppel TD, Muller S, et al. SPECT/CT with ${ }^{99 m}$ Tc-MAA in radioembolization with ${ }^{90} \mathrm{Y}$ microspheres in patients with hepatocellular cancer. J Nucl Med. 2009;50:688-692.

12. Ahmadzadehfar H, Sabet A, Biermann K, et al. The significance of ${ }^{99 \mathrm{~m}} \mathrm{Tc}-\mathrm{MAA}$ SPECT/CT liver perfusion imaging in treatment planning for ${ }^{90} \mathrm{Y}$-microsphere selective internal radiation treatment. J Nucl Med. 2010;51:1206-1212.

13. SIR-Spheres microspheres [package insert]. North Sydney, NSW, Australia: Sirtex Medical Limited; May 2016.
14. Therasphere microspheres [package insert]. Farnham, Surrey, U.K.: Biocompatibles UK Ltd.; Rev. 14, 2017.

15. Willowson K, Bailey DL, Baldock C. Quantitative SPECT reconstruction using CT-derived corrections. Phys Med Biol. 2008;53:3099-3112.

16. Ritt P, Vija H, Hornegger J, Kuwert T. Absolute quantification in SPECT. Eur J Nucl Med Mol Imaging. 2011;38(suppl 1):S69-S77.

17. Bailey DL, Willowson KP. An evidence-based review of quantitative SPECT imaging and potential clinical applications. J Nucl Med. 2013;54:83-89.

18. Yu N, Srinivas SM, Difilippo FP, et al. Lung dose calculation with SPECT/CT for ${ }^{90}$ yttrium radioembolization of liver cancer. Int J Radiat Oncol Biol Phys. 2013; 85:834-839.

19. Kao YH, Magsombol BM, Toh Y, et al. Personalized predictive lung dosimetry by technetium-99m macroaggregated albumin SPECT/CT for yttrium-90 radioembolization. EJNMMI Res. 2014;4:33.

20. Bernardini M, Smadja C, Faraggi M, et al. Liver selective internal radiation therapy with ${ }^{90} \mathrm{Y}$ resin microspheres: comparison between pre-treatment activity calculation methods. Phys Med. 2014;30:752-764.

21. Gulec SA, Mesoloras G, Stabin M. Dosimetric techniques in ${ }^{90}$ Y-microsphere therapy of liver cancer: the MIRD equations for dose calculations. J Nucl Med. 2006;47:1209-1211.

22. Armstrong J, Raben A, Zelefsky M, et al. Promising survival with three-dimensional conformal radiation therapy for non-small cell lung cancer. Radiother Oncol. 1997;44:17-22.

23. Karimi R, Tornling G, Forsslund $\mathrm{H}$, et al. Lung density on high resolution computer tomography (HRCT) reflects degree of inflammation in smokers. Respir Res. 2014;15:23.

24. Gates GF, Orme HW, Dore EK. Measurement of cardiac shunting with technetium-labeled albumin aggregates. J Nucl Med. 1971;12:746-749.

25. Sabet A, Ahmadzadehfar H, Muckle M, et al. Significance of oral administration of sodium perchlorate in planning liver-directed radioembolization. J Nucl Med. 2011;52:1063-1067.

26. De Gersem R, Maleux G, Vanbilloen H, et al. Influence of time delay on the estimated lung shunt fraction on ${ }^{99 \mathrm{~m}} \mathrm{Tc}$-labeled MAA scintigraphy for ${ }^{90} \mathrm{Y}$ microsphere treatment planning. Clin Nucl Med. 2013;38:940-942.

27. Grosser OS, Ruf J, Kupitz D, et al. Pharmacokinetics of ${ }^{99 \mathrm{~m}}$ Tc-MAA- and ${ }^{99 \mathrm{~m}} \mathrm{Tc}-$ HSA-microspheres used in preradioembolization dosimetry: influence on the liver-lung shunt. J Nucl Med. 2016;57:925-927.

28. Theysohn JM, Schlaak JF, Muller S, et al. Selective internal radiation therapy of hepatocellular carcinoma: potential hepatopulmonary shunt reduction after sorafenib administration. J Vasc Interv Radiol. 2012;23:949-952.

29. Ahmadzadehfar H, Sabet A, Meyer C, Habibi E, Biersack HJ, Ezziddin S. The importance of Tc-MAA SPECT/CT for therapy planning of radioembolization in a patient treated with bevacizumab. Clin Nucl Med. 2012;37:1129-1130.

30. Ward TJ, Tamrazi A, Lam MG, et al. Management of high hepatopulmonary shunting in patients undergoing hepatic radioembolization. J Vasc Interv Radiol. 2015;26:1751-1760.

31. Kokabi N, Camacho JC, Xing M, et al. Open-label prospective study of the safety and efficacy of glass-based yttrium 90 radioembolization for infiltrative hepatocellular carcinoma with portal vein thrombosis. Cancer. 2015;121:2164-2174.

32. Gaba RC, Zivin SP, Dikopf MS, et al. Characteristics of primary and secondary hepatic malignancies associated with hepatopulmonary shunting. Radiology. 2014;271:602-612.

33. Elschot M, Nijsen JF, Lam MG, et al. ${ }^{99 \mathrm{~m}} \mathrm{Tc}-\mathrm{MAA}$ overestimates the absorbed dose to the lungs in radioembolization: a quantitative evaluation in patients treated with ${ }^{166}$ Ho-microspheres. Eur J Nucl Med Mol Imaging. 2014;41:19651975 . 